\title{
Workflow disruptions and surgical performance: past, present and future
}

Douglas A Wiegmann, ${ }^{1}$ Thoralf M Sundt ${ }^{2}$

'Department of Industrial and Systems Engineering, University of Wisconsin-Madison, Madison, Wisconsin, USA

${ }^{2}$ Division of Cardiac Surgery, Massachusetts General Hospital, Massachusetts, USA

\section{Correspondence to} Dr Douglas A Wiegmann, Department of Industrial and Systems Engineering, University of Wisconsin-Madison, Madison, Wisconsin, USA; dawiegmann@wisc.edu

Accepted 8 January 2019 Published Online First 25 January 2019

\section{Sinked}

- http://dx.doi.org/10.1136/ bmjqs-2018-007957

\section{Check for updates}

(c) Author(s) (or their employer(s)) 2019. No commercial re-use. See rights and permissions. Published by BMJ.

To cite: Wiegmann DA, Sundt TM. BMJ Qual Saf 2019;28:260-262.
The earliest contributions of human factors engineering to surgery probably occurred just over a century ago. Already well-known for their pioneering scientific approaches to management, Frank and Lillian Gilbreth turned their expertise in time and motion studies and the psychology of management to optimising the operating room environment (and various aspects of hospital operations). ${ }^{1}$ Some readers may know this real husband and wife pair of early management gurus as the parents depicted in 'Cheaper by the Dozen'. Written by two of their 12 children, it humorously depicted the application of the science of efficiency to the lives of this family of 14 . The book, first published in 1946, generated a film (1950), a television series and an updated film in 2003. None of the antics in these various productions convey the real-life contributions of either of the Gilbreths. For instance, the modern approach to laying out surgical instruments in regular and consistent patterns grew out of their detailed analyses of filmed surgeries, in which the Gilbreths noticed that surgeons often spent more time searching for their instruments than they did operating. ${ }^{1}$

Within contemporary circles, however, many would likely cite de Leval and colleagues $^{2}$ as the first to empirically demonstrate the relationship between human factors in the operating room (OR) and surgical error. They studied surgical performance and outcomes across a series of 243 arterial switch operations performed by 21 surgeons. One of their most notable findings pertains to the role that system factors play in the aetiology of errors. Such factors were generally referred to as 'minor' events and included such factors as failed alarm systems, coordination problems with the blood bank, perfusion issues and inappropriate task delegation. These minor events tended to disrupt to the flow of a surgical procedure, which subsequently impacted negatively the performance of even experienced surgical teams. Specifically, as the number of minor events increased, the ability of a surgical team to cope with major problems decreased significantly. ${ }^{34}$ The accumulation of minor events appeared to diminish the compensatory resources of the surgical team, increasing their vulnerability and susceptibility to committing errors. $^{56}$

Although de Leval et al, clearly delineated the types of 'minor' events that they observed specifically, there remained a paucity of information about the underlying nature and frequency of minor events that occur in the OR more broadly. As a result, generalisable approaches to remediating these 'minor' events were not immediately evident. Consequently, in the early 2000 s, our research team, ${ }^{78}$ as well as other researchers in Europe, ${ }^{9} 10$ set out to further explore the exact nature and frequency of minor events in the $\mathrm{OR}$ and their potential impact on overall surgical performance.

Based on our interdisciplinary understanding of surgical performance and human factors engineering, we began studying and documenting factors in the OR that could impede the progress of a case. ${ }^{7}$ We named these factors 'surgical flow disruptions' (SFDs) and developed a process for categorising them based on a human factors approach. ${ }^{8}$ Our initial categories included technological issues, environmental distractions, teamwork/ miscommunication, training delays and resource availability. We then correlated the frequency in which each type of SFD occurred with our other observations of performance during surgical cases. We found an overall positive correlation 
between the rate in which different SFD occurred and the rate of errors that transpired during a case.

The findings of our initial study helped to further reinforce the notion that even experienced surgical teams can be impacted by the context in which they work. While such a conclusion may seem obvious to many, the prevailing culture within the field of surgery at the time focused almost exclusively on surgeon skill. ${ }^{10}$ When errors occurred, the surgeon was the one to 'blame'. Actions taken to remediate the problem therefore often included admonishment or retraining. However, our empirical findings that no surgeon is an island, capable of achieving excellence independent of their team or OR setting, further opened the door for human factor engineers and surgeons to work together to focus on systems issues rather merely training and skill development. James Reason's adage that 'we cannot change the human condition, but we can change the conditions under which people work ${ }^{11}$ had now gained considerable traction.

Since our first publication in this area, numerous studies have been conducted by our research team and others to further explore SFDs during surgery. These studies have highlighted the fact that SFDs are not always the same across institutions or types of surgical cases. Other studies have expanded this line of research to explore SFDs (often referred to as simply 'flow disruptions' (FDs)) across a diverse range of surgical procedures and healthcare settings. ${ }^{12-14}$ Other studies have expanded on our methodology to develop standardised SFD data collection and analysis tools. ${ }^{15}$ Indeed, it now appears that the study of observable and quantifiable flow disruptions that potentially impact patient care is becoming a common approach to improving quality and safety.

One of the most exciting advancement in this line of research is the growing sophistication in how SFD are analysed to better understand their interrelationships and actual impact on performance. ${ }^{16}$ To date, the vast majority of research in this area has simply focused on describing the nature of different SFDs and the frequency of in which they occur. Yet, not all SFDs are likely to impact performance in the same way. Some researchers have found that breakdowns in teamwork and communication are most impactful on overall surgical performance, ${ }^{8}$ whereas others have found that OR layout ${ }^{17}$ is highly influential. Furthermore, SFDs do not appear to be independent of one another. The findings by de Leval and colleagues $^{2}$ clearly suggest that SFDs can interact in a way that fosters subsequent SFDs and that SFDs can have a cascading effect, which increases their potential impact on performance.

The study by Joseph et al in this issue of BMJ Quality and Safety ${ }^{18}$ provides an excellent example of how the study and analysis of SFDs can be advanced. These authors analysed video recordings from four digital cameras located in the four corners of the
OR (the Gilbreths would be proud!). This method allowed for a more systematic identification of SFDs and their temporal relationships in a manner not typically afforded by simple observational methods and hand-written notes. The authors were able to identify a total of 2504 disruptions across 28 surgeries. They also adapted a methodology proposed by Parker et $a l^{15}$ for scoring the severity of each disruption in terms of its actual impact on performance. The severity of each disruption was classified into one of six categories $(1-$ no impact/minor disruption, no response; 2-momentary disruption (acknowledgement of disruption, no pause in task); 3-momentary distraction (short pause $<10 \mathrm{~s}$ ); 4-primary task interrupted (task cessation $>10 \mathrm{~s}$ ); 5-primary task disruption (secondary task engaged) and 6repeat task. For the purposes of further analysis, the authors then defined any SFD that resulted in a pause or break in the primary activity as a 'major flow disruption' (categories 3-6). All other SFDs were designated as 'minor flow disruptions' (categories 1 and 2).

Joseph et al were able to develop logistic and linear regression models that allowed them to explore possible relationships between minor and major SFDs. Results revealed that increases in layout-related minor SFDs significantly increased the rate of major SFDs and that there was a significant association between minor SFDs related to equipment/ furniture positioning and the rate of major SFDs. The study contains numerous other findings related to the roles of staff members involved in SFDs, types of staff activities associated SFDs and OR traffic-related factors. As noted by the authors, this study is, to date, the 'most comprehensive attempt to observe and model FDs and their systemic causes'. Indeed, the findings of the study are too numerous to summarise here. So, we strongly recommend those interested in this topic to read the study by Joseph and colleagues.

In closing, we would like to note that the main reason for studying SFDs and their interrelationships is not simply to understand them. Rather, it is to use our understanding of SFDs so that we can reduce their occurrence or mitigate their consequences when they do occur. Nevertheless, studying SFDs using sound methodologies and robust analytical methods is a crucial step in identifying appropriate interventions. The growing body of literature clearly indicates that the types of SFDs that impact performance vary across contexts. Hence, no single solution is likely to work across all settings or even address all the types of SFDs that may occur. Given the limited resources, the study of SFD affords the ability to judiciously and systematically invest in improving safety and patient care. Furthermore, a mature process for measuring and analysing SFDs also provides the means for empirically evaluating whether any intervention has been effective in achieving one's desired goals. ${ }^{19-21}$ 
There are likely to be instances where certain interventions prove to have an impact while others do not. A reliable means of evaluating the efficacy of our efforts, or return on investments, thus constitutes the vital key to progress.

Funding The authors have not declared a specific grant for this research from any funding agency in the public, commercial or not-for-profit sectors.

Competing interests None declared.

Patient consent Not required.

Provenance and peer review Commissioned; internally peer reviewed.

\section{REFERENCES}

1 Baumgart A, Neuhauser D, Frank ND. Frank and Lillian Gilbreth: scientific management in the operating room. Qual Saf Health Care 2009;18:413-5.

2 de Leval MR, Carthey J, Wright DJ, et al. Human factors and cardiac surgery: a multicenter study. J Thorac Cardiovasc Surg 2000;119:661-72.

3 Reason J. Human error: models and management. BMJ 2000;320:768-70.

4 Reason J. Heroic compensations: the benign face of the human factor. Flight Safety Australia 2001;5:29-31.

5 Carthey J, de Leval MR, Reason JT. The human factor in cardiac surgery: errors and near misses in a high technology medical domain. Ann Thorac Surg 2001;72:300-5.

6 Carthey J, de Leval MR, Wright DJ, et al. Behavioural markers of surgical excellence. Saf Sci 2003;41:409-25.

7 ElBardissi AW, Wiegmann DA, Dearani JA, et al. Application of the human factors analysis and classification system methodology to the cardiovascular surgery operating room. Ann Thorac Surg 2007;83:1412-9.

8 Wiegmann DA, ElBardissi AW, Dearani JA, et al. Disruptions in surgical flow and their relationship to surgical errors: an exploratory investigation. Surgery 2007;142:658-65.

9 Healey AN, Primus CP, Koutantji M. Quantifying distraction and interruption in urological surgery. Qual Saf Health Care 2007;16:135-9.
10 C V, Morrthy K, Sarker SK. Systems approaches to surgical quality and safety from concept to measurement. Ann Surg 2004;239:475-82.

11 Reason J. Managing the risks of organizational accidents. Aldershot UK: Ashgate, 1997.

12 Catchpole KR, Gangi A, Blocker RC, et al. Flow disruptions in trauma care handoffs. J Surg Res 2013;184:586-91.

13 Duff S, Boquet A, Kring J. Identification and classification of flow disruptions in the operating room during two types of general surgery procedures. Proceedings of the $54^{\text {th }}$ Annual Meeting of the Human Factors and Ergonomics Society. Santa Monica, CA: HFES, 2010.

14 Schemmel A, Lee M, Hanley T, et al. Radiology workflow disruptors: a detailed analysis. J Am Coll Radiol 2016;13:1210-4.

15 Parker SEH, Laviana AA, Wadhera RK, et al. Development and evaluation of an observational tool for assessing surgical flow disruptions and their impact on surgical performance. World $J$ Surg 2010;34:353-61.

16 Shao X, Zhong X, Li J, et al. Bottleneck analysis to reduce surgical flow disruptions: theory and application. IEEE Trans Autom Sci Eng 2015;12:127-39.

17 Cohen TN, Cabrera JS, Sisk OD, et al. Identifying workflow disruptions in the cardiovascular operating room. Anaesthesia 2016;71:948-54.

18 Joseph A, Khoshkenar A, Taaffe KM, et al. Minor flow disruptions, traffic-related factors and their effect on major flow disruptions in the operating room. BMJ Qual Saf 2019;28:276-83.

19 Wadhera RK, Parker SH, Burkhart HM, et al. Is the "sterile cockpit" concept applicable to cardiovascular surgery critical intervals or critical events? The impact of protocol-driven communication during cardiopulmonary bypass. J Thorac Cardiovasc Surg 2010;139:312-9.

20 Catchpole K, Ley E, Wiegmann D, et al. A human factors subsystems approach to trauma care. JAMA Surg 2014;149:962-8.

21 Henrickson SE, Wadhera RK, Elbardissi AW, et al. Development and pilot evaluation of a preoperative briefing protocol for cardiovascular surgery. J Am Coll Surg 2009;208:1115-23. 